\title{
Analyse Critique d'une Politique d'Assistance aux Réfugiés: Les Sites d'Installations Agricoles Ouverts pour les Réfugiés Érythréens au Soudan
}

\author{
Shoa Asfaha et Véronique Lassailly-Jacob
}

\section{Introduction}

La question des réfugiés est un sujet brûlant de l'actualité africaine. On compte aujourd'hui environ 6 millions de réfugiés en Afrique dont 750000 au Soudan (World Refugee Survey, 1993), un chiffre largement sous-estimé puisqu'il ne prend en compte qu' une partie des fugitifs, ceux qui sont enregistrés et qui répondent aux critères définis par la Convention de Genève et par celle de l'Organisation de l'Unité Africaine (OUA). Ne sont pas inclus tous ceux qui trouvent refuge à l'intérieur de leur propre pays: plus de 5 millions de Soudanais sont des "réfugiés de l'intérieur." Ne sont pas inclus non plus tous ceux qui ont franchi une frontière et se sont fondus clandestinement dans le milieu rural ou dans les villes du pays d'accueil, bien souvent les plus nombreux.

Le Soudan, à l'exemple de nombreux pays africains, est a la fois un pays producteur de réfugiés et un pays d'asile:

Sudan-Africa's largest countrycontinued to produce as well as host massive numbers of uprooted persons during 1992. At year's end, some five million Sudanese were internally displaced by civil war and natural disasters, 263,000 Sudanese were refugees outside their country, and about 750,000 refugees from neighbouring countries resided in Sudan (World Refugee Survey 1993, 73).

Les 750000 réfugiés recensés au Soudan en 1992 provenaient d'Érythrée,
d'Éthiopie, du Tchad, de l'Ouganda et du Zaïre. Parmi eux, on comptait environ 530000 Errythréens dont 280000 regroupés dans des sites d'installations encadrés et 250000 spontanément installés en ville ou dans des villages (World Refugee Survey 1993, 62).

Cet article évoque la situation des réfugiés érythréens regroupés dans des sites d'installations agricoles dans l'est du Soudan et s'interroge sur le bien-fondé de cette politique d'assistance coûteuse: ces sites d'installations sont-ils en mesure d'intégrer économiquement et socialement les réfugiés dans le pays d'accueil pendant la durée de leur exil? Cet article souligne tout d'abord l'originalité juridique et administrative de la politique d'asile soudanaise ainsi que la diversité et la générosité des programmes d'assistance aux réfugiés, appliqués grâce à la collaboration de la communauté internationale et de multiples ONG. Il met ensuite l'accent sur les obstacles que rencontrent les réfugiés encadrés dans ces sites pour devenir autosuffisants et localement intégrés et enfin pose la question du devenir de ces sites et de la région d'accueil lorsque les réfugiés seront rentrés chez eux.

\section{Contexte juridique et administratif de la politique d'asile Soudanaise}

David Smock, évoquant la situation des Érythréens au Soudan, souligne:

The most notable feature of Sudan's response is hospitality. No Eritrean has been turned back at the border

\footnotetext{
Shoa Asfaha est Érythréenne et socio-économiste. Elle a séjourné à plusieurs reprises au Soudan où elle a mené des enquêtes dans les sites d'installations agricoles ouverts pour les réfugiés érythréens. Les résultats de ses enquêtes ont fait l'objet de sa thèse de doctorat soutenue en 1988 d̀ l'Ecole des Hautes Études en Sciences Sociales d Paris. Elle est actuellement membre de Care Britain d Londres comme coordonnatrice des programmes de développement en Afrique de.l'Est et du Sud.

Véronique Lassailly-Jacob est française et gégraphe, chargée de Recherches au C.N.R.S. (Centre National de la Recherche Scientifique) dans un laboratoire de Sociologie et Géographie Africaines. Elle est actuellement accueillie comme "Visiting Scholar" au Centre de Recherche sur les Réfugiés da l'Université York a Toronto.
}

and, once inside the Sudan, no refugee has been forcibly repatriated $-\mathrm{a}$ striking contrast to many other countries, particularly in Asia, which repulse refugees at the border or which expel those who have managed to enter (Smock 1982, 455).

Comme dans la plupart des sociétés africaines, l'hospitalité est une des grandes valeurs traditionnelles de la société soudanaise. Le Soudan a une longue tradition d'accueil de populations venues d'Égypte, d'Abyssinie, $d^{\prime}$ Arabie, d'Afrique Occidentale et Centrale, se déplaçant pour des raisons économiques, religieuses ou politiques. Les flux de populations les plus connus sont les réfugiés Coptes chassés d'Égypte au VIIème et VIIİ̀me siècle, les groupes de pèlerins musulmans se rendant au lieu saint du Mahdi pour obtenir sa bénédiction ou bien de passage vers La Mecque ou encore la fuite des Haoussa du Nord du Nigéria face à l'occupation Britannique au début du siècle, et enfin celle des migrants saisonniers venus du Tchad et de l'Érythrée. Les mouvements d'exode contemporains, c'est-à-dire les flux de réfugiés s'intègrent dans ce cadre historique des migrations vers le Soudan.

Les grandes lignes de la politique $d^{\prime}$ asile soudanaise s'inspirent des principes de la Convention de Genève (1951), du protocole des Nations Unies de 1967, et de la Convention de l'OUA (1969) relatifs au statut des réfugiés. Bien avant de ratifier en 1972 ces traités juridiques internationaux et régionaux, le Soudan poursuivait déjà une politique de porte ouverte "open door policy" à l'égard des réfugiés: le terme légal de "réfugie" fut officiellement adopté en 1967 lorsque fut créé le Commissariat Soudanais d'Aide aux Réfugiés (COR), cinq ans avant la ratification de ces traités.

En 1974, le Soudan se dotait d'une juridiction nationale concernant le 
droit d'asile (Regulation of Asylum Act) et l'article 44 de sa constitution reconnut les droits des réfugiés à demander l'asile politique en conformité avec les principes internationaux et les lois nationales. Rappelons que la définition du réfugié adoptée par la juridiction soudanaise est plus large que celle des Conventions de Genève et de l'OUA, "le terme Réfugié s'applique aussi aux orphelins de guerre et à ceux dont les tuteurs ont disparu."

Ces textes reflètent la prise de conscience précoce de la question des réfugiés et l'originalité de la politique $\mathrm{d}^{\prime}$ asile soudanaise. Le Soudan est un des rares pays $d^{\prime}$ Afrique, avec la Tanzanie, qui se soient dotés $d^{\prime}$ instruments juridiques nationaux de protection des réfugiés.

Les structures administratives d'assistance aux réfugiés dans de nom- breux pays africains sont généralement représentées par des instances gouvernementales déjà en place et des organisations non-gouvernementales (ONG). Le Soudan a été un des seuls pays africains à avoir créé une structure spécifique (COR) pour gérer et dispenser l' aide aux réfugiés. Le COR, interlocuteur principal de la commu. nauté internationale, a la responsabilité de la mise en oeuvre du programme d'assistance. Le "Refugee Fund Bill" de 1980 lui donna toute autorité pour gérer l'assistance internationale. Placé à l'origine sous la tutelle du Ministère de l'Intérieur, son rôle et ses activités ont pris une telle ampleur en raison de l'accroissement constant du nombre des réfugiés au cours des années, qu'il apparaît aujourd'hui comme un Ministère à part entière.

\section{La politique d'assistance soudanaise}

Face à un afflux croissant de réfugiés de longue durée, -le conflit érythréen a duré près de 30 ans-, le Commissariat Soudanais d'Aide Aux Réfugiés fit appel à la communauté internationale (le HCR, Haut Commissariat des Nations Unies pour les Réfugiés, le PAM, Programme Alimentaire Mondial, l'UNICEF, Fonds des Nations Unies pour l'Enfance, le BIT, Bureau International du Travail), ainsi qu'à un grand nombre d' ONG nationales ou internationales, pour l'aider à accueillir ces réfugiés et leur fournir des conditions de vie décentes.

Le Soudan adopta une politique d'asile "généreuse" et un programme d'assistance extrêmement ambitieux en décidant d'accueillir le plus grand

\section{Abstract}

\section{A Critical Analysis of a Refugee Aid Policy: The Agricultural Settlements Established for Eritrean Refugees in the Sudan}

This paper highlights the impact of the Sudanese assistance policy on the Eritrean refugees regrouped in UNHCR-sponsored agricultural settlements in Kassala province, in eastern Sudan. The pursuit of self-sufficiency through agriculture, local integration and the future prospects of the settlements are the main issues discussed. First, the singularity of the Sudanese legal, administrative and aid policy toward refugees is discussed Sudan has always adopted an "open door policy" toward migrants and refugees, and has a long tradition of hospitality. It has not only ratified the various UN instruments governing refugee status but also has its own Asylum Act which is one of the most liberal documents of its kind, and has created a permanent administrative body through which assistance is provided to refugees.

Second, the paper outlines the long history of Eritrean migration to Sudan. Since the beginning of the century, Eritrean farmers and herdsmen have seasonally crossed the borders in search of agricultural work or pasture for their cattle. The Ethio-Britrean war is recalled as the major cause of contemporary refugee movements since the mid-1960s. The pattern of refugee settlement - spontaneous versus organized-is briefly discussed. The organized agricultural settlements have been the focus of the assistance policy, as they are considered to be the best option in promoting refugee self-sufficiency and local integration in the host community.
Third, the paper points out the special features of these agricultural settlements and assesses the obstacles which prevent refugees from becoming self-sufficient through agriculture. While refugees are provided with plots of land, tools and seeds to become self-supporting. their means of survival come from other activities which develop informally. The concept of organized settlement and the way refugees are perceived by others have become one of the major obstacles preventing local integration. It is Kassala Province rather than the refugee settlements which has benefited the most from the international community's refugee assistance. Kassala has also benefited from cheap refugee labour used to develop its infrastructure, in particular the agricultural sector.

Finally, the issue of sustainability of the organized settlements is raised, particularly in light of the onset of the spontaneous return of refugees to their homeland. What will be the impact of this important labour force's departure on the province's economy? Will the Sudanese government be able to maintain the infrastructure and facilities in the organized settlements? While many research programs are undertaken on reintegration problems of the refugees in their homeland, too little attention has been paid to the impact of refugee departure and particularly the future of the organized settlements which have been planned and managed at high cost. 
nombre de réfugiés dans des sites d'installations encadrées de différents types. Toutefois, cette politique d'assistance ne s'appliqua qu' aux réfugiés enregistrés, c'est-à-dire ceux établis dans des sites d'installations encadrées. Non seulement les réfugiés clandestinement installés ne reçurent presqu'aucun support et assistance mais encore ceux qui vivaient en ville firent l'objet de tracasseries administratives, d'arrestations et même de déportations vers des sites encadrés. La politique d'assistance soudanaise chercha à décourager toute implantation spontanée des réfugiés, en particulier dans les villes.

Grâce aux concours technique et financier du HCR, quatre types d' installations encadrées furent mis en place: camps de réception, sites d'installations agricoles, sites d'installations rurales basées sur le salariat agricole et sites d'installations semi-urbaines. Le Soudan est le seul pays à avoir cré un si grand nombre (plus de 70) et un tel éventail d'installations encadrées. Le modèle le plus fréquent et qui fit l'objet d'infrastructures coateuses fut celui des sites agricoles où des terres arables furent distribuées aux familles afin qu'elles atteignent une certaine autosuffisance et s'intègrent dans la société d'accueil pendant la durée de leur exil.

Les camps de réception ou centres d'accueil provisoires furent ouverts non loin des frontières pour accueillir et enregistrer les nouveaux arrivés. Ils y reçurent une aide d'urgence -nourriture, abri, soins médicaux- qui leur fat fournie par des organisations humanitaires. Les réfugiés devaient séjourner provisoirement dans ces camps, entre 6 mois et un an, avant d'être transférés dans des sites d'installations aménagés à l'intérieur du pays; en réalité, le gouvernement et le HCR ne disposèrent pas de moyens financiers et logistiques suffisants leur permettant de créer suffisamment de sites pour absorber toute la population réfugiée résidant dans les camps. C'est pourquoi des milliers de réfugiés séjournèrent plus de 10 ans dans ces camps provisoires. On peut imaginer les répercussions de ce séjour de totale dépendance sur leur attitude future. Néanmoins, les transferts vers les sites encadrés, ouverts à l'intérieur du Soudan, ont souvent été impopulaires et ont parfois donner lieu à des résistances, les réfugiés préférant rester non loin de la frontière, esperant ainsi retourner plus facilement et plus rapidement chez eux dès que la situation le leur permettrait.

Les sites $d^{\prime}$ installations agricoles ${ }^{1}$ eurent pour objectif de créer des communautes économiquement viables et localement intégrées afin de les faire contribuer au développement national et d'alleger ainsi le fardeau de l'accueil. Des financements considérables ont été alloués pour mettre en place à partir de 1969 ce programme d'assistance dans l'est et le sud du Soudan. Les premiers sites furent ouverts dans la zone de Semsem près du centre urbain de Quala-En-Nahal. Ainsi, furent crés d'une part 24 sites d'installations agricoles à l'est du pays qui hébergeaient près de 130000 réfugiés érythréens et éthiopiens et d'autre part, 47 sites ${ }^{2}$ au sud du pays pour environ 160000 réfugiés ougandais et quelques milliers de Zaïrois (Stein and Clark 1990, 4). Les réfugiés y reçurent des exploitations agricoles de 2 à 4 ha, des outils, des semences et les prestations de labour d'un service de tracteurs. L'assistance internationale est dispensée dans ces sites jusqu'à ce qu'ils deviennent économiquement viables, $l$ 'estimation de viabilité étant basée sur le niveau de vie des populations locales; les réfugiés ne doivent pas bénéficier de conditions de vie meilleures que celles des autochtones. Lorsque ce stade est atteint, après une période d'environ 3-4 ans, le HCR doit se retirer et transférer ses compétences au gouvernement soudanais qui prend la relève de la gestion de l'installation. Lelotissement est alors intégré dans les structures administratives du Soudan.

Les sites d'installations rurales basées sur le salariat agricole ou "wageearning settlements" sont un type d'installation développé surtout au Soudan. Ces sites ont été ouverts à proximité des grands périmètres irrigués de Al-Suki (non loin de la Gezirah), la Nouvelle Halfa et Rahad.
Les réfugiés regroupés dans ces sites ne reçoivent pas de terres agricoles et doivent survivre en se salariant dans les périmètres irrigués d'État et dans les grandes exploitations agricoles privées ou étatiques. Il existe 6 sites de ce type hébergeant environ 30000 personnes.

Les sites d'installations semi-urbaines sont situés à la périphérie des grandes villes de l'est du pays et ont été conçus pour des réfugiés d'origine urbaine. L'espoir du gouvernement était de pouvoir regrouper progressive, ment tous les réfugiés urbains dans ces sites afin de réduire les pressions qu'ils exerçaient en ville sur les équipements collectifs et le logement. De nombreux micro-projets, surtout artisanaux et générateurs de revenus, furent mis en place dans ces sites par des ONG. Toutefois, d'une part ces micro-projets ne correspondaient pas toujours aux attentes des réfugiés urbains-plusieurs sites ont même été désertés- d'autre part, l'État et le HCR n'ont pas eu les moyens financiers et techniques pour creer des sites suffisamment attractifs et nombreux.

\section{Réfugiés érythréens au Soudan}

La migration érythréenne vers le Soudan n'est pas un fait nouveau. Au début du siècle, agriculteurs et éleveurs érythréens traversaient périodiquement la frontière soudanaise, les premiers pour s'employer comme salariés agricoles, les seconds pour faire transhumer leurs troupeaux. Ce va-et-vient saisonnier, accepté de part et d'autre, se transforma complètement à partir des années 60 . Le conflit politico-militaire, aggravé par les rivalités entre les superpuissances, qui opposa les nationalistes érythréens au régime éthiopien, ${ }^{3}$ transforma dès 1967, un mouvement migratoire saisonnier en un mouvement de refuge. Des motivations migratoires d'ordre économique devinrent essentiellement politiques et écologiques (la sécheresse dévastatrice de 1984-85 précipita les mouvements de population vers le Soudan); une migration saisonnière se transforma en une migration durable; une migration d'agriculteurs et d'éleveurs 
se gonfla d'une population urbaine; enfin, une migration spontanée et dont la destination était du seul ressort de l'intéressé devint une migration forcée, souvent encadrée par des instances nationales et même internationales jusque sur des sites imposés par les autorités du pays d'accueil. Ce sont tous ces caractères spécifiques qui ont transformé ces migrants en réfugiés.

Les premiers flux importants de réfugiés érythréens au Soudan, soit près de 30000 personnes, datent de 1966-67. Ils furent suivis de plusieurs importants mouvements de fugitifs en 196970 (50 000 personnes), en $1975-76$ (50 000 personnes dont 20000 s'installerent dans le camp de réception de Wad El Hilau), en 1977-78 (plus de 200000 personnes, en grande partie des réfugiés urbains), en 1982 (plus de 10000 personnes) et en 1984-85 (près de 200 000 personnes à nouveau). Depuis le début des années 60 , près de 600000 réfugiés érythréens ont trouvé refuge au Soudan. Certains ont continué leur route vers un pays tiers dans le cadre de "resettlement programmes" (en Europe, aux USA, au Canada), les autres, la quasi-totalité, sont restés au Soudan. Leur intégration locale dans la société soudanaise s'est produite de façon spontanée ou encadrée, en milieu rural comme en milieu urbain.

L'installation spontanée en milieu rural a surtout été le fait de la première vague de migration lorsque les fugitifs par petits groupes traversèrent la frontière la plus proche et se réfugièrent dans les villages et hanieaux situés près de la frontière soudanaise. Les premiers groupes de réfugiés érythréens qui arrivèrent au Soudan vers le milieu des années soixante venaient surtout des Basses Plaines érythréennes; beaucoup d'entre eux appartenaient aux communautés Maria, Beni-Amer et Bedja et avaient des membres de leur famille ethnique au Soudan. Ils pratiquaient depuis longtemps la transhumance au Soudan oriental avant que la guerre puis la sécheresse ne les obligent à chercher refuge de l'autre côté de la frontière. Aussi, furent-ils pris en charge spontanément par ceux des leurs qui rési-

Figure 1: Sites d'Installations Agricoles dans l'Est du Soudan

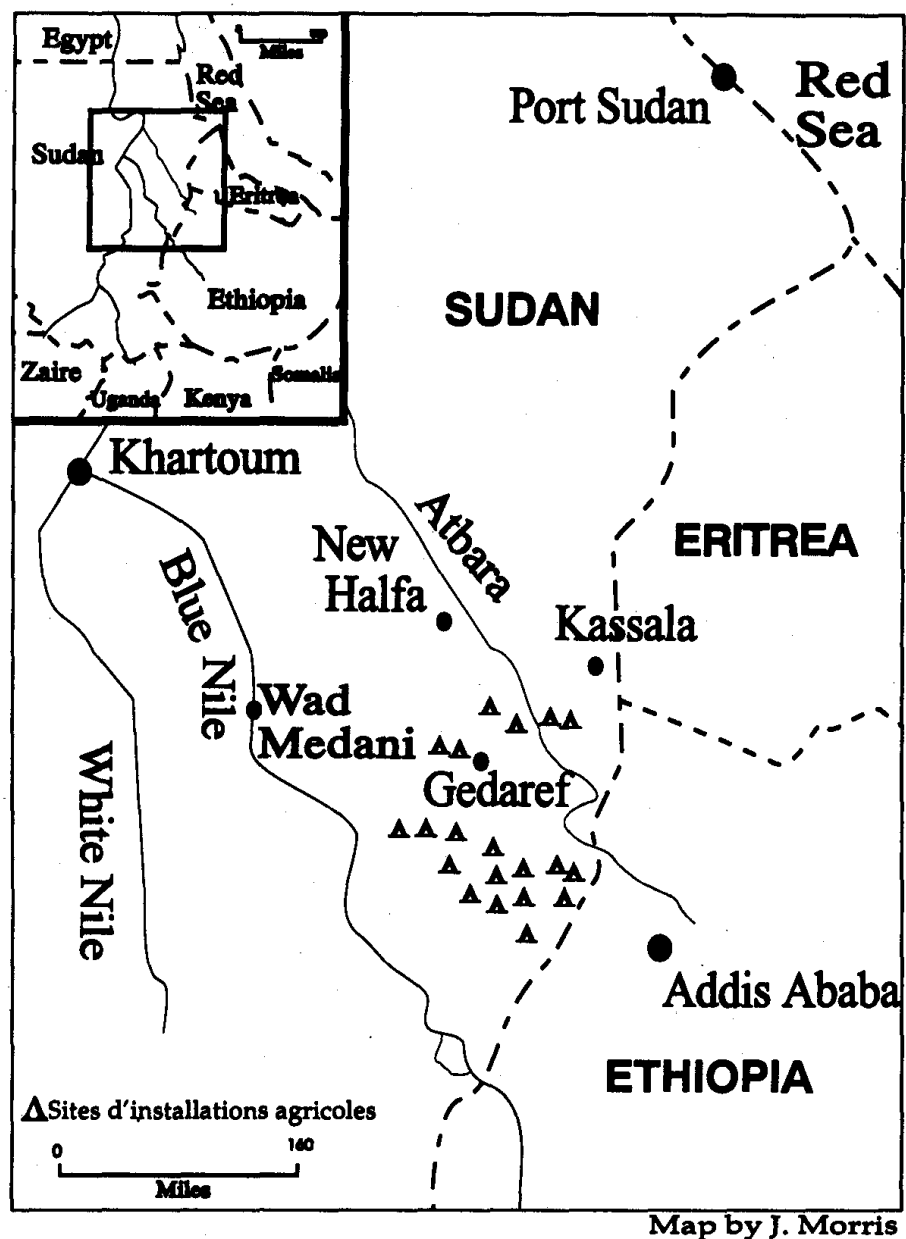

daient au Soudan. Ces réfugiés s'installèrent clandestinement dans les villages et hameaux de leurs hôtes et partagèrent leurs services et leurs ressources. Pendant longtemps, leur nombre et leur situation matérielle furent ignorés des pouvoirs publics car ces réfugiés ne reçurent aucune attention de la part des institutions d'aide. Pour avoir une vague estimation de leur nombre, il faudra attendre 198485 , lorsque la gravité de la sécheresse contraindra de nombreux réfugiés "spontanés" à se rendre jusqu'aux camps deréception et dans les sites d'installations pour se faire enregistrer afin d' obtenir des rations alimentaires.

De nombreux réfugiés érythréens d'origine urbaine se sont également installés spontanément dans les villes du Soudan oriental et à Khartoum. A partir des années 80 , la politique de "Terreur rouge" et l'encadrement forcé par le régime du Dergue d'Éthiopie força de nombreux jeunes à fuir les villes d'Erythrée et d'Ethiopie. Le Soudan est un des rares pays Africains à avoir accueilli un si grand nombre de réfugiés urbains. Ces réfugiés refusent généralement d'être encadrés et regroupés; ils préfèrent chercher individuellement des solutions à leur survie dans les grandes villes soudanaises. En 1988, on estimait à près de 230000 , le nombre des réfugiés urbains installés à Port-Soudan, Kassala, Gédaref et Khartoum. Pour décourager leur concentration dans les villes, l'État et les 
organismes d'assistance ne leur apportent pratiquement aucune aide et la police procède périodiquement à des rafles, organisées soi-disant pour contrôle d' identité.

La situation au Soudan oriental commença à se détériorer lorsque le mouvement des fugitifs s'amplifia et que la pression sur les services publics et sur les ressources (terres arables, eau potable et bois de feu en particulier) de la région d'accueil devint telle que la compétition s'installa entre populations locales et nouveaux venus pour l'accès à ces ressources. Des relations de fraternité clanique se transformèrent peu à peu en relations entre population hôte et étrangers. Les tensions furent d'autant plus vives que les nouvelles vagues de réfugiés, les Tigrigna, les Saho, provenaient de régions plus éloignées, celles des Hauts Plateaux érythréens où les populations n'avaient plus de liens ethniques et d'affinités culturelles avec leurs hôtes. C'est alors que s'organisa l'installation encadrée par le gouvernement soudanais et le HCR.

Environ 87000 réfugiés érythréens ont été regroupés dans 19 sites d'installations, sites agricoles ou sites basés sur le salariat, et localisés dans la province de Kassala. Ces populations réinstallées ne représentent qu'une minorité des réfugiés érythréens (près de $15 \%$ du nombre total estimé de réfugiés érythréens) et appartiennent à différentes ethinies. ${ }^{4}$ Les autres réfugiés vivent soit dans des camps de réception, soit parmi les populations locales dans les villes importantes (Khartoum, Wad Medani, Gedaref, Port Sudan, Kassala, etc.) et dans les villages et hameaux de la province de Kassala.

\section{Réfugiés érythréens dans les sites d'installations agricoles}

Un site $d^{\prime}$ installation agricole se présente sous la forme d'un gros lotissement aux formes géométriques, entouré de terres arables. Il bénéficie en général d'un niveau élevé d'infrastructures tel que approvisionnement en eau potable ${ }^{5}$, routes d'accès, dispensaires, écoles, centres communautaires, lieux de culte, etc. Le HCR finança en grande partie ces infrastructures et prit en charge les frais de déplacement des réfugiés depuis les camps d'accueil jusqu' aux sites. A son arrivée, chaque chef de famille reçoit un lot de $200 \mathrm{~m}^{2}$ et des subsides pour construire son habitation, le "tukul." 6

Les "tukul" sont rangés le long d'un réseau quadrillé de rues. Un site d'installation agricole se différencie $d^{\prime} u n$ camp par le niveau élevé de ses infrastructures et équipements sociaux (dont les locaux sont généralement construits "en dur") et par la présence d'un terroir agricole. Il se différencie également d'un village car les familles qui y résident ne sont pas apparentées et ne disposent pas d'une même organisation sociale et culturelle. Un site est constitué de différents quartiers dans lesquels les réfugiés sont répartis en fonction de leur appartenance ethnique. Il rassemble une population qui varie entre 3000 et 6000 personnes et la distance au terroir agricole peut atteindre 5 a $10 \mathrm{~km}$. Par son type d'urbanisme et sa taille, un site d'installation agricole est plus apparenté à un lotissement urbain en milieu rural qu'à un village.

Le programme agricole fût le volet central de l'assistance fournie aux réfugiés dans les sites. Chaque chef de famille reçat l'usage d'une exploitation ou "hawasha" de 5 à 10 "feddan" (un "feddan" équivaut à 0,42ha) soit 2,1 à 4,2 ha, des semences de sorgho ainsi que des outils. Le sorgho ou "durra", aliment de base de la population locale, est la monoculture pratiquée dans les sites les plus septentrionaux. Dans la partie méridionale de la province de Kassala, là où le climat est plus favorable, des semences de sésame ont également été distribuées comme culture de rente. Un service de location de tracteurs fut mis en place dans tous les nouveaux sites pour assurer les prestations de labour. Pendant les deux premières années de leur séjour dans l'installation, les familles reçurent des rations alimentaires $d u$ PAM. Toutefois, de nombreux obstacles ont contribué à freiner la viabilité économique et l'intégration locale de ces sites $d^{\prime}$ installations agricoles.

\section{Obstacles à l'autosuffisance économique}

\section{Des localisations defavorables}

La plupart des sites implantés dans $l^{\prime}$ Est du Soudan se trouvent dans la partie semi-désertique de la province de Kassala. Les terres y sont de potentialités agricoles médiocres et cette zone est plus propice à un élevage transhumant qu'à une agriculture sous pluie très risquée; Une vie sédentaire agricole n'y est possible qu' avec l'aide de l'irrigation car les pluies sont irrégulières et insuffisantes: la pluviosité moyenne varie entre 400 et $600 \mathrm{~mm}$ et ne permet qu'une courte saison culturale de 3 à 4 mois. Or, l'irrigation n'a pas été développée pour les réfugiés. De plus, le choix des sites n'a pas fait l'objet d'études préalables sérieuses; Prospections pédologiques, reconnaissance des ressources en eau ont été menées à la hâte quant elles n'ont pas été inexistantes. Plusieurs sites ont même été retenus par le gouvernement en dépit des avertissements de non viabilité donnés par les missions de prospection internationales (Stein and Clark 1990).

Le rôle et la responsabilité des autorités du pays d'accueil dans le choix des sites sont primordiaux. Le gouvernement soudanais n'a pas tenu compte des critères climatiques, pédologiques, économiques et sociaux établis par le HCR pour le choix des sites d'installation. Contrairement au discours officiel qui soutient que le choix des sites $d$ 'installations repose en premier lieu sur l'intérêt des réfugiés, on constate que les motivations qui ont guidé le gouvernement soudanais dans sa sélection des sites sont essentiellement d'ordre économique et géopolitique: économique, en établissant ces sites à proximité des grands périmètres irrigués d'État et des grandes exploitations pluviales privées ou étatiques afin qu'ils disposent de la maind'oeuvre réfugiée; géopolitique, en dispersant ces sites afin de respecter un certain équilibre démographique avec la population locale et d'éviter une effervescence politique erythréenne. 
Des exploitations de taille insuffisante

L'exploitation familiale a été retenue comme unité de production agricole. Le HCR et le COR avaient déclaré qu'une exploitation de 10 "feddan" serait le seuil de superficie minimum permettant l'auto-subsistance d'une famille moyenne. Or, les exploitations distribuées sont fréquemment de taille inférieure à 10 "feddan" en raison de terroirs agricoles trop restreints. De plus, l'exiguité des superficies des exploitations ne permet pas de pratiquer un système de jachère, pratique courante chez les exploitants soudanais de la zone, en vue de régénérer la fertilité du sol. L'absence de jachère dans les exploitations des réfugiés entraîne une baisse des rendements et une dégradation des sols.

\section{Un calendrier de labour mal organisé}

Le labour des parcelles a lieu au début de la saison des pluies et précède le semis. Il est effectué grâce à un service de location de tracteurs, les exploitants payant un prix forfaitaire peu élevé. Ce service a également été étendu aux petits exploitants soudanais qui ont leurs champs à proximité de ceux des réfugiés, ceci dans le but de créer une bonne entente entre les deux communautés. La date du labour détermine en grande partie le succès de la campagne agricole. Or, les retards sont fréquents dûs en partie à des pannes, manque de carburant ou de pièces de rechange; mais ces retards sont surtout causés par le détournement de ce service vers les exploitations de grands fermiers soudanais qui, payant le prix comptant, ont la priorité sur les réfugiés et les petits exploitants locaux. Les retards de la prestation de labour entraînent la chute des rendements culturaux.

\section{Manque de main-d'oeuvre familiale}

Si le labour est mécanisé, les autres activités agricoles sont entièrement manuelles et la main-d'oeuvre occupe une place importante dans ces activités (deux sarclages, coupe des épis, battage et mise en sac des grains). En général, les hommes opèrent seuls ou bien sont aidés par leurs fils mais ce travail familial estinsuffisant. Les femmes des groupes Baria, Cunama, Tigrigna et Bilein, qui, en Érythrée, participaient à la vie économique et sociale de leur village, notamment aux travaux des champs aux côtés de leurs époux, ne le peuvent plus dans les sites agricoles. En effet, les communautés ethniques qui interdisent à la femme d'exercer des activités économiques en dehors du foyer conjugal, comme les Beni-Amer et les Maria, dominent très souvent l'espace culturel et social des installations. Ces groupes ethniques majoritaires vont dominer l'espace culturel, social et économique du nouvel habitat et imposer leurs pratiques, étouffant celles des ethnies minoritaires. La position sociale de la femme dans cette société multiculturelle de réfugiés constitue un exemple remarquable illustrant ce phénomène.

Pour faire face à ce manque de maind'oeuvre familiale ou à une maind'oeuvre familiale inexpérimentée pour les travaux agricoles (cas des nombreux réfugiés d'origine nomade), pour également réduire la durée du temps de récolte afin d'éviter les ravages des animaux des éleveurs de la zone, de nombreux exploitants doivent avoir recours à une maind'oeuvre salariée. Or, les rendements agricoles sont souvent trop faibles pour à la fois nourrir les familles et payer les coûts d'une main-d'oeuvre salariée. Aussi, l'agriculture n'assure pas les besoins de base des familles réfugiées.

La faiblesse des rendements de sorgho et le caractère saisonnier du travail agricole oblige les cultivateurs à pratiquer d'autres activités. Ils vont se salarier dans les périmètres irrigués d'État et dans les grandes fermes privées, ou bien pratiquer l'élevage et le petit commerce. On remarque que de nombreux sites d'installations agricoles ne sont pas devenus économiquement viables à travers le programme agricole mis en place par l'assistance.

\section{Obstacles à l'intégration locale}

Cette intégration locale est freinée par de nombreux obstacles que l'on peut identifier comme la conception des sites, leur planification et le statut des réfugiés.

\section{La conception des sites}

Des communautés ethniques diverses qui, dans le pays d'origine, évoluaient chacune dans leur propre espace géographique, économique, social et culturel, tout en entretenant entre elles des relations d'échanges multiples, sont amenées à cohabiter dans un même espace géographique restreint qui est le site d'installation. Ces familles, de filiation patriarcale ou matriarcale, de confession musulmane, chrétienne ou animiste, de tradition agricole, nomade ou urbaine, doivent apprendre à vivre ensemble quotidiennement puisqu'elles fréquentent les mêmes marchés, points d'eau, écoles, dispensaires, clubs sociaux. Cette cohabitation forcée même si elle est parfois l'objet de tensions et de conflits, atténue peu à peu les barrières socioculturelles et les préjugés ethniques qui existaient auparavant entre ces différentes communautés. Elle favorise même un renforcement des liens de solidarité entre membres de différentes ethnies. Le fait d'être des "Ladjiin" (qui veut dire réfugiés) pousse les familles à s'entraider moralement et économiquement pour faire face à la dureté de leur vie d'exil. Un "ladji" qui doit être hospitalisé en ville, va bénéficier de l'aide de toute la communauté rêfugiée, toutes ethnies et religions confondues.

Cette solidarité apprend aux réfugiés à dépasser leur conscience ethnique pour développer une conscience nationale. Ce sentiment de conscience nationale se développe aussi par diverses manifestations comme des chants "révolutionnaires". Ces chants sont l'expression métaphorique de l'unité et véhiculent l'idée d'une patrie qui était perdue mais qui sera bientôt retrouvée. Pour les enfants qui ont grandi en exil et qui chantent bras dessus-bras dessous, le message transmis par ces chants est immense et le concept d'identité nationale devient peutêtre plus concret et plus réel pour eux que pour leurs parents. 
Bien que les réfugiés doivent s'adapter à un environnement socioculturel nouveau, celui de la société de la région d'accueil, -ils côtoient les populations soudanaises habitant dans les environs, les nomades arabes, Shukriya ou Ja'aliyiin ou encore les "Westerners", migrants venus autrefois d'Afrique de l'Ouest-, en vivant dans les sites, ils ont moins de contacts avec la société du pays d'accueil que leurs compatriotes résidant parmi les populations locales. On remarque que l'intégration linguistique est souvent mieux réalisée par les réfugiés spontanément installés. Tels sont les résultats des interviews de Bulcha (1987) auprès de réfugiés éthiopiens dans l'Est du Soudan, qui révèlent que $82 \%$ des réfugiés spontanément installés parlent l'arabe contre $48 \%$ seulement dans les sites d'installation.

\section{La planification des sites}

De nombreux indices relatifs à la planification de ces sites dénotent leur caractère provisoire et statique et sont autant de freins à leur integration locale. Tout $d$ 'abord, ces sites n'ont pas de nom propre. Ils reçoivent un nom local, le nom d'un village soudanais voisin. Ensuite, les réfugiés n'ont pas de droit de propriété mais un droit d'usufruit sur leurs lopins de terre. Enfin, ces installations sont conçues de façon statique. Seule la population nouvellement arrivée est prise en compte pour la distribution des lots et des exploitations. Les planificateurs n'ont pas considéré les besoins d'espaces supplémentaires nécessaires à la croissance naturelle de la population et au déclin éventuel de la fertilité du sol. Celui qui vient de se marier doit construire son propre "tukul" à l'intérieur de l'enclos familial et cultiver une partie de l'exploitation de son père, ce qui entraîne une forte densification de l'habitat et un morcellement croissant des exploitations.

\section{Le statut des réfugiés}

Le facteur le plus important qui freine l'intégration d'un réfugié dans son pays d'accueil, quelque soit son mode $d$ 'installation et son niveau de vie, est son statut légal. La possibilité pour les réfugiés de longue durée de devenir citoyens du pays d'accueil est une exception. ${ }^{7}$ Avoir un statut de réfugié signifie être soumis à des lois spéciales. Selon la législation soudanaise (Regulation of Asylum Act 1974), les réfugiés n'ont pas de droits politiques (droit de vote), ne peuvent quitter le site d'installation sans autorisation écrite (titre de voyage obligatoire) ${ }^{8}$, ne peuvent posséder des biens immobiliers ou fonciers et ne peuvent obtenir de permis de travail en dehors du site. Il est même souvent difficile pour ces derniers d'obtenir un permis de travail pour d'autres activités ou une licence pour faire du commerce. Aussi, beaucoup de réfugiés ne peuvent exercer leur métier par manque de permis de travail. Toutes ces atteintes aux libertés du réfugié lui donnent le sentiment qu'il est en quelque sorte "un prisonnier" dans l'installation.

\section{Conclusion}

Il est sâr que les sites d'installations agricoles ont contribué à atténuer les effets traumatisants de l'exil pour des milliers de réfugiés et leur ont donné les moyens d'atteindre une certaine autosuffisance. Grâce aux équipements sociaux, centres de santé, écoles primaires et autres infrastructures, les réfugiés connaissent une amélioration de leurs conditions de vie même si la viabilité de ces équipements demeure incertaine.

Cependant, ces sites d'installations agricoles n'ont pas atteint leurs objectifs d'autosuffisance et d'intégration locale à travers les programmes mis en place:

- D'autosuffisance au moyen du programme agricole imposé, car les récoltes et les revenus tirés de l'agriculture sont trop faibles. Si les réfugiés survivent dans ces sites, c'est grâce à des activités non encadrées qu'ils entreprennent spontanément et parallèlement à l'agriculture. Le programme agricole, pilier de l'assistance, apparaît donc comme une façade qui permet surtout de légitimer la présence de certains organismes donateurs comme le HCR. Et promouvoir un tel programme agricole ne fait qu' alourdir l'administration des sites et grève énormément les fonds de l'assistance.

- D'intégration locale, car rassemblés dans ces sites, les réfugiés vont conserver leur identité de réfugié, renforcer leurs liens de solidarité, entretenir leur désir de retour et cultiver leur différence avec la société d'accueil. Les éléments "fondamentaux" de l'identité des réfugiés vont se conserver et se perpétuer dans ces lotissements ruraux qui, par leur existence même, sont un frein à l'intégration locale dans la société d'accueil.

Il semblerait que le choix des localisations des sites par le gouvernement soudanais ait surtout été guidé par le souci à la fois de constituer une réserve de main-d'oeuvre agricole bon marché disponible pour l'agriculture soudanaise et de développer la région orientale du Soudan par toutes les infrastructures et les équipements qui y ont été implantés grâce à l'aide internationale. Ce que voulait le Soudan, c'est le partage du fardeau de l'accueil avec la communauté internationale et une assistance qui profite au développement régional du pays par les infrastructures mises en place.

Depuis mai 1991, date de la libération totale de l'Érythrée, on assiste à un phénomène de retour spontané des populations réfugiées érythréennes vers leur pays d'origine. Le gouvernement érythréen a mis en place une structure administrative appelée Commission for Eritrean Refugee Affairs (CERA) qui, en collaboration avec des agences des Nations-Unies et des ONG, est chargée du programme de rapatriement et de réintégration des réfugiés. Ce programme en trois phases consiste à rapatrier et à réintégrer dans leur société d'origine environ 500 000 réfugiés érythréens résidant au Soudan. Bien que ce programme ait été approuvé par les différentes institutions concernées, son financement $n$ 'a pas encore été assuré et l'accord tripartite entre le $\mathrm{HCR}$, le gouvernement 
soudanais et le gouvernement erythréen concernant le rapatriement des populations n'a pas encore été signé, ce qui retarde le démarrage d'un vaste rapatriement encadré. Toutefois, ce blocage administratif et financier n'a pas empêché un mouvement de retour déjà bien entamé. Près de 100000 réfugiés érythréens sont rentrés spontanément, certains bénéficiant $d$ 'un minimum d'aides alimentaire et logistique fournies par le CERA et autres organisations caritatives locales, les autres se débrouillant par eux-mêmes. La question de la réintégration de ces populations qui, pour certaines, ont vécu plus de 20 ans en exil, fait l'objet de vastes programmes de recherche et accapare l'attention de la communauté internationale et d'une grande partie du monde académique travaillant sur le problème des réfugiés.

Toutefois, l'impact du départ de ces milliers de personnes sur l'économie du pays d'accueil est une recherche encore négligée. Qui va remplacer cette main-d'oeuvre "bon marche" qui faisait "tourner" les grands périmètres irrigués et les plantations de la province orientale? Quel sera l'avenir de ces sites agricoles, de leurs infrastructures, équipements, terres défrichées et labourees? Les populations soudanaises locales vont-elles bénéficier de ces sites équipés et aménagés? Mais surtout, le gouvernement soudanais sera-t-il en mesure d'entretenir et de faire fonctionner les infrastructures et équipements? Réintégration des populations érythréennes dans leur région d'origine mais aussi conséquences de leur départ sur l'économie de la province de Kassala, sont des grandes recherches qui doivent désormais être menées.

\section{Notes}

1 Les installations agricoles font partie d'une nouvelle politique d'assistance qui relève plus d'une aide au développement que $d^{\prime} u n e$ stricte action humanitaire d'urgence. Inaugurée en Afrique par le HCR et largement développee sur ce continent depuis les années 60 -le Soudan, la Tanzanie, 1'Ouganda, le Zaire, le Botswana, le Burundi et la Zambie sont aujourd'hui les principaux pays d'asile ayant adopté cette forme d'assistance aux réfugiés- elle a été ensuite étendue à l'Amé- rique Latine et $\mathrm{l}^{\prime}$ Asie du Sud-Est mais a une echelle bien moindre. On trouve quelques exemples d'installations agricoles a Belize, au Panama, au Costa Rica, au Honduras, Nicaragua et en Malaisie. En Afrique, le Soudan a crét le plus grand nombre de sites (plus de 70) et la Tanzanie est le pays qui a le mieux intégré ces sites dans sa politique de développement agricole et son programme de "villagisation". Depuis 1961, plus de 140 sites d'installations agricoles furent crés en Afrique, principalement en Afrique de l'Est et du Sud. Environ un million de réfugiés seulement en Afrique ont été concernés par cette forme d'assistance. D'un coté, le coat de ces installations ne permet pas de les multiplier à une grande échelle, de l'autre, nombreux sont les refugiés qui refusent de vivre dans ces lotissements trop encadrés et controlés et qui prefèrent se cacher en ville ou en brousse. Enfin, quelques gouvernements comme celui de l' Afrique du Sud, s'opposent à cette forme d'assistance jugte comme une solution trop durable.

2 Les 47 "villages" de réfugiés ouverts au Sud Soudan furent fermés en 1988-89 quand les réfugiés retournèrent en Ouganda.

3 Colonie italienne à partir de 1890, l'Érythrée fut ensuite occupé par les Britanniques en 1940-41 qui l'administrèrent jusqu'en 1952, date a laquelle l'Érythrée devint, par décision des Nations-Unies, féderée à l'Éthiopie. En septembre 1961, la guerre de liberation débuta. En Novembre 1962, l'empereur Hailé Sélassié procéda a l'annexion pure et simple de l'entité autonome d'Érythrée qu'il déclara province éthiopienne. 1974 marqua la déposition du Négus après 50 ans de règne, l'arrivée au pouvoir du Dergue et la liberation temporaire des villes érythréennes par les deux fronts érythréens (F.P.L.E., Front Populaire de Liberration de l'Erythrée et F.L.E., Front de Libération de l'Erythrée). 1978 fut l'anné de la grande offensive du Dergue, soutenue par I'URSS, qui entraîna une généralisation de la guerre dans toute l'Érythrée et la reprise des villes par l'armée éthiopienne. Cette guerre de liberation ("Opération Red Star" est la dernière grande offensive éthiopienne lancée en 1982), aggravée par la terrible sécheresse de 1984-85 et la lutte interne entre les differents fronts, causèrent des pertes humaines considérables et la fuite des populations par dizaines de milliers. En mai 1991, l'Érythrée fut liberée par le FPLE et, à la suite d'un référendum organisé sous l'égide des Nations-Unies, l'Érythrée accéda à son indépendance le 24 mai 1993.

4 En Érythree, cohabitent 9 groupes ethniques, ayant chacun sa propre identité culturelle, linguistique et religieuse, ainsi que son propre mode de vie. Ils se répartissent entre les Hauts-Plateaux, les plaines cotières et les plaines de l'intérieur. Les agriculteurs sédentaires représentent environ $50 \%$ de la population et sont établis en grande partie sur les Hauts-Plateaux. Ce sont les groupes Tigrigna, Saho, Cunama, Baria et Bilein. Les éleveurs nomades forment environ $30 \%$ de la population, transhument principalement dans les Basses Plaines et forment les groupes Tigré composés de plusieurs grandes tribus comme les Beni-Amer, les Maria, le groupe Bedja, le groupe Afar, et le groupe Rashaida. Environ $20 \%$ de la population totale habite les villes, situées principalement dans la région des Hauts-Plateaux (a l'exception de Massawa) et habitées majoritairement par les Tigrigna et les Bilein. Les principales ethries réfugiées au Soudan sont les Tigrigna, Tigre, Baria, Saho, Bilein et Cunama.

5 Un système très sophistique d'adduction d'eau équipé de pompes a été installe dans de nombreux sites, dont ceux de Quala EI Nahal. En 1974, il y eut transfert des sites d' installations de Quala en Nahal au gouvernement soudanais mais celui -ci n'a pas pu assurer le fonctionnement de ces sites. In n'y avait plus de pizces de rechange et de carburant pour les tracteurs et les pompes défectueuses n'assuraient plus I'approvisionnement en eau des sites. Lorsque plus de la moitie de la population fut contrainte de quitter ces sites, le gouvernement soudanais fit de nouveau appel au HCR et a d'autres organismes pour reprendre leur assistance.

6 Le "tukul", est l'habitation traditionnelle de cette région du Soudan, une case généralement ronde, d'une seule piece, dont les murs sont constitués d'une armature en bois colmatée de terre battue et dont le toit est en paille tressée. La grandeur du "tukul" traduit la taille de la famille et la prosperité de celle-ci.

7 Quelques Beni-Amer érythréens résidant en ville ont réussi à etre naturalisés, aidés par des partis politiques soudanais dans un but électoral.

8 Etant donne les lenteurs administratives, le candidat est souvent oblige d'attendre plusieurs semaines pour obtenir une autorisation de déplacement quil n'est valable que pour un seul déplacement.

\section{Bibliographie}

Asfaha, S. 1988. "De l'Érythrée au Soudan: Contribution geographique a l'étude d'une politique d'assistance aux réfugiés." Thèse de Doctorat. Paris: Ecole des Hautes Etudes en Sciences Sociales.

1992. "Un espace agraire loti au Soudan: Les sites d'installation des réfugiés Erythréens." Espace Geographique 21(4):347356.

Balamoan, A. 1976. "Peoples and Economics in the Sudan, 1884-1956." Cambridge: Harvard University Center for Population Studies.

Bascom, J.B. 1989. "Social Differentiation among Eritrean Refugees in Eastern Sudan: The Case of Wad el Hileau." Joumal of Refugee Studies 2(4):403-418.

Bulcha, M. 1987. "Sociological and Economic Factors in Refugee integration: The Case of Ethiopian Exiles in the Sudan." In Refugees and Development in Africa, edited by P. Nobel. Uppsala: Scandinavian Institute of African Studies. 